\title{
Wavelet 2D Modal Curvature for Identifying Non-Uniform Cracks in Plates under Noisy Conditions
}

\author{
Wei XU11, Mao-Sen $\mathrm{CAO}^{1, \text { a }}$, Yu-Feng ZHANG ${ }^{2,3}$ and Maciej Radzieński ${ }^{4}$ \\ 1Department of Engineering Mechanics, Hohai University, 210098 Nanjing, China \\ 2Jiangsu Transportation Institute Group Co., Ltd., 211112 Nanjing,China \\ ${ }^{3}$ National Key Laboratory for Safety and Health of Long-span Bridge in Service, 211112 Nanjing, \\ China \\ ${ }^{4}$ Institute of Fluid-Flow Machinery, Polish Academy of Sciences, 80-231 Gdansk, Poland \\ acmszhy@hhu.edu.cn
}

Keywords: Damage detection; Non-uniform crack; Plate; Modal curvature; Wavelet transform; Noise interference.

\begin{abstract}
Identifying non-uniform cracks in plates is a challenging problem worthy of exploration. 2D modal curvature is often utilized to detect damage in plates, but its capability to precisely identify non-uniform cracks has not been resolved well, largely due to two noticeable deficiencies, lack of sensitivity to damage and susceptibility to noise interference. With this concern, this study ameliorates the 2D modal curvature using the wavelet transform, whereby the wavelet $2 \mathrm{D}$ modal curvature is constructed. Damage features extracted from the wavelet 2D modal curvature can be employed to identify non-uniform cracks in plates. This wavelet $2 \mathrm{D}$ modal curvature enjoys the multiscale analysis characteristics introduced by the wavelet transform, leading to synchronous extraction of damage features and elimination of noise interference. These features endow the wavelet 2D modal curvature with the capability to identify non-uniform cracks under noisy conditions. The performance of the wavelet 2D modal curvature is verified by damage detection in a plate with a non-uniform crack. The results show the wavelet $2 \mathrm{D}$ modal curvature can effectively identify non-uniform cracks in plates under noisy conditions.
\end{abstract}

\section{Introduction}

After long-term operation of structures, incipient small-scale damage can accumulate and develop to large-scale damage, finally threatening the safety of the structures [1,2]. Study of structural damage detection is therefore of great significance in science and engineering and has become a research focus during the past decades in the fields of mechanical, aerospace, and civil engineering [3-7]. Plate-like structural components are essential elements in many structures. Among the types of damage in plate-like structures, cracking is the most common. Most related research has focused on identifying cracks. However, little attention has been paid to characterizing the trend of change in depth or the weakening of the material properties along the cracks, ignoring the fact that in reality most cracks are non-uniform. Hence, characterizing non-uniform cracks is a challenging problem worthy of exploration. With this motivation, this study proposes a method for identifying nonuniform cracks, with the emphasis on characterization of non-uniformity.

Among the methods for damage detection in plate-like structures, the two-dimensional modal curvature $\left(\mathrm{MC}^{2 \mathrm{D}}\right)$-based method has been widely investigated over the last two decades [8]. In proposing a new method, this study begins with the $\mathrm{MC}^{2 \mathrm{D}}$. Representative studies of $\mathrm{MC}^{2 \mathrm{D}}$-based damage identification in plates are summarized as follows. Yam et al. [9] created a damage index using the difference between a pair of $\mathrm{MCs}^{2 \mathrm{D}}$ for intact and damaged plates. The capability of this index to characterize damage was demonstrated on cracked aluminum plates. Qiao et al. [10] employed the $\mathrm{MC}^{2 \mathrm{D}}$ method to detect delamination in a composite laminate. The presence, location, and size of the delamination were effectively detected. Li et al. [11] developed two damage indices using $\mathrm{MCs}^{2 \mathrm{D}}$, namely a bending moment index and a residual strain mode shape index, utilizing 
them to identify damage in a free-boundary plate. The results showed that damage indices could locate the damage accurately. Hu et al. [12] evaluated the $x-$ and $y-$ directional $\mathrm{MCs}^{2 \mathrm{D}}$ of a plate by individually differentiating a mode shape in the $x$ - and $y-$ directions, and utilized these $\mathrm{MCs}^{2 \mathrm{D}}$ to locate delamination in plates. Numerical results showed that sudden changes in the $\mathrm{MCs}^{2 \mathrm{D}}$ correctly designated the region of the delamination. Wu and Law [13] utilized the curvature of a uniform load surface resembling a $\mathrm{MC}^{2 \mathrm{D}}$ to indicate damage in plates, and the location of the damage was accurately identified. Yoon et al. [14] ameliorated the $\mathrm{MC}^{2 \mathrm{D}}$ method by the 2D gapped smoothing method (2D GSM) and utilized it to inspect plates for damage. The numerical and experimental results indicated that the method was capable of detecting damaged regions in plates. Yang et al. [15] proposed the Fourier spectral $\mathrm{MC}^{2 \mathrm{D}}$ method, and the method was proved to be effective in detecting damage in composite plates.

Despite the prevalence of the $\mathrm{MC}^{2 \mathrm{D}}$ method in identifying damage in plate-like structures, direct use of the method for damage detection in plates is hindered by its two noticeable deficiencies, lack of sensitivity to incipient small-scale damage and susceptibility to noise interference [16]. Addressing these two deficiencies, researchers in recent years have made efforts to improve the $\mathrm{MC}^{2 \mathrm{D}}$ method: Yoon et al. [14] proposed the 2D GSM to reveal small changes in the $\mathrm{MC}^{2 \mathrm{D}}$ caused by the damage, by which small damage can be correctly located. Yang et al. [15] utilized the 2D Fourier transform rather than second-order differentiation to produce the $\mathrm{MC}^{2 \mathrm{D}}$, by which noise interference could be effectively suppressed. Recently, by ameliorating the $\mathrm{MC}^{2 \mathrm{D}}$ with the wavelet transform (WT) and Teager energy operator (TEO), Xu et al. [16] proposed an integrated method and successfully utilized it to identify the presence, location, and shape of an X-shaped notch in a plate with the aid of the non-contact measurement. Although a great deal of effort has been expended to deal with the two deficiencies of the $\mathrm{MC}^{2 \mathrm{D}}$ method, accurate extraction of small-scale damage features in the $\mathrm{MC}^{2 \mathrm{D}}$ under noise conditions is still a challenge of the method.

With this concern, this study addresses ameliorating the $\mathrm{MC}^{2 \mathrm{D}}$ by the WT, by which the wavelet $\mathrm{MC}^{2 \mathrm{D}}$ (including the RW (real wavelet)-MC $\mathrm{MC}^{2 \mathrm{D}}$ and the $\mathrm{CW}$ (complex wavelet)-MC ${ }^{2 \mathrm{D}}$ ) are presented progressively, allowing features to be extracted to identify non-uniform cracks in plate-like structures, with the emphasis on characterization of non-uniformity. This new wavelet $\mathrm{MC}^{2 \mathrm{D}}$ benefits from the characteristics of the multiscale analysis introduced by the WT, and can be utilized to for synchronous extraction of damage features and elimination of noise interference. Therefore, the new wavelet $\mathrm{MC}^{2 \mathrm{D}}$ features are considered capable of identifying non-uniform cracks under noise conditions.

\section{Wavelet $M^{2 D}$ Features for Identifying Non-Uniform Cracks}

This section addresses ameliorating the $\mathrm{MC}^{2 \mathrm{D}}$ by the $\mathrm{WT}$, by which the wavelet $\mathrm{MC}^{2 \mathrm{D}}$ (including the $\mathrm{RW}-\mathrm{MC}^{2 \mathrm{D}}$ and the $\mathrm{CW}-\mathrm{MC}^{2 \mathrm{D}}$ ) is presented progressively, allowing features to be extracted to identify non-uniform cracks in plate-like structures, with the emphasis on characterization of nonuniformity.

\section{$\mathrm{MC}^{2 \mathrm{D}}$}

The $\mathrm{MC}^{2 \mathrm{D}}$ of a plate at $\left(x_{0}, y_{0}\right)$ is expressed as the sum of the partial derivatives of the mode shape $W$ in the $x$ - and $y$-directions, denoted as $\nabla^{2} W[13,14,16]$ :

$$
\nabla^{2} W\left(x_{0}, y_{0}\right)=\left.\left[\frac{\partial^{2} W(x, y)}{\partial x^{2}}+\frac{\partial^{2} W(x, y)}{\partial y^{2}}\right]\right|_{\substack{x=x_{0} \\ y=y_{0}}}
$$

Damage in plate-like structures can cause change in the $\mathrm{MC}^{2 \mathrm{D}}$; in turn, modification of the $\mathrm{MC}^{2 \mathrm{D}}$ can manifest the presence of damage in the plate. The $\mathrm{MC}^{2 \mathrm{D}}$ method is a non-baseline method, that is, with no requirement for baseline information of structures, such as materials, geometry, and boundary conditions. Besides, the $\mathrm{MC}^{2 \mathrm{D}}$ possesses the property of isotropy, which means that it is capable of identifying cracks with arbitrary directions in plate-like structures [16]. 
Rather than directly using the sum of the partial derivatives to represent the $\nabla^{2} W$ by Eq. (13), in this study, an alternative method is introduced to equivalently represent the $\mathrm{MC}^{2 \mathrm{D}}$ more simply: first, the second-order derivatives at $\left(x_{0}, y_{0}\right)$ in the $x$ - and $y$-directions are calculated by rows and columns, along $y=y_{0}$ and $x=x_{0}$, respectively [12]; then the results in the $x$ - and $y$-directions are totalled:

$$
\begin{aligned}
\nabla^{2} W\left(x_{0}, y_{0}\right) & =\left.\left[\frac{\partial^{2} W(x, y)}{\partial x^{2}}+\frac{\partial^{2} W(x, y)}{\partial y^{2}}\right]\right|_{\substack{x=x_{0}, y=y_{0}}} \\
& =\left.\frac{\partial^{2} W(x, y)}{\partial x^{2}}\right|_{\substack{x=x_{0} \\
y=y_{0}}}+\left.\frac{\partial^{2} W(x, y)}{\partial y^{2}}\right|_{\substack{x=x_{0}, y=y_{0}}} \\
& =\left.\frac{d^{2} W\left(x, y_{0}\right)}{d x^{2}}\right|_{\substack{x=x_{0}\\
}}+\left.\frac{d^{2} W\left(x_{0}, y\right)}{d y^{2}}\right|_{\substack{y=y_{0}\\
}}
\end{aligned}
$$

This equivalent calculation procedure of the $\mathrm{MC}^{2 \mathrm{D}}$ is advantageous for simplifying the calculation of the RW-MC ${ }^{2 \mathrm{D}}$ and the $\mathrm{CW}-\mathrm{MC}^{2 \mathrm{D}}$ presented in the following parts.

\section{Wavelet MC $^{2 \mathrm{D}}$}

As the WT is linear, the manner of calculating the $\mathrm{MC}^{2 \mathrm{D}}$ can be applied to the RW-MC ${ }^{2 \mathrm{D}}$ and the $\mathrm{CW}-\mathrm{MC}^{2 \mathrm{D}}$ linearly transformed from the $\mathrm{MC}^{2 \mathrm{D}}$, by which merely the $1 \mathrm{D} \mathrm{WT}$ are required for the RW-MC ${ }^{2 \mathrm{D}}$ and the CW-MC ${ }^{2 \mathrm{D}}$, with no need to use $2 \mathrm{D} \mathrm{WT}$ involving more complex calculation.

By ameliorating the $\mathrm{MC}^{2 \mathrm{D}}$ with the wavelet, the $\mathrm{MC}^{2 \mathrm{D}}$ expressed in Eq. (14) is transformed to the wavelet $\mathrm{MC}^{2 \mathrm{D}}$ at $u=x_{0}$ and $v=y_{0}$ :

$$
\begin{aligned}
W_{s}^{*}(u, v) & =s^{2} \frac{d^{2} W\left(u, y_{0}\right) \otimes \bar{g}_{s}(u)}{d u^{2}}+s^{2} \frac{d^{2} W\left(x_{0}, v\right) \otimes \bar{g}_{s}(v)}{d v^{2}} \\
& =W\left(u, y_{0}\right) \otimes \bar{\psi}_{s}(u)+W\left(x_{0}, v\right) \otimes \bar{\psi}_{s}(v)
\end{aligned}
$$

where $u$ and $v$ are defined as translation parameters in the $x$ - and $y$-directions, respectively, $s$ is the scale parameter, $g(x)$ is the function to generate the mother wavelet, $\psi(x)=(-1)^{2} \frac{d^{2} g(x)}{d x^{2}}$, and $\bar{\psi}_{s}(x)=\frac{1}{\sqrt{s}} \psi\left(\frac{-x}{s}\right)$. The essential relationship between Eq. (3) and Eq. (2) is that $W$ in Eq. (2) is replaced with the convolution of $W$ and the window $\bar{g}_{s}$ in the $x$ - and $y$-directions, respectively. As the additional scale parameter $s$ is introduced, the multiscale analysis of the WT for structural damage detection is established [17]: when the scale increases, noise interference can be eliminated while damage-caused singularity can be retained to characterize damage features, and the presence and location of damage can be identified by the singularity peak.

\section{Method Verification}

To verify the method, an aluminum rectangular plate simply supported in its four edges is taken as a specimen. The dimensions of the plate are length $a=180 \mathrm{~mm}$, width $b=270 \mathrm{~mm}$, and $h=4 \mathrm{~mm}$ in the $x$-, $y$-, and $z$-directions, respectively. The Young's modulus, material density, and Poisson's ratio are $70.5 \mathrm{Gpa}, 2680 \mathrm{~kg} / \mathrm{m}^{3}$, and 0.33 , respectively. Depicted in dimensionless coordinates, a horizontal (in $x$-direction) crack $10 \mathrm{~mm}$ in length (spanning from $\zeta_{1}=0.2$ to $\zeta_{2}=0.3,2 c=0.1$ ) and 0.4 $\mathrm{mm}$ in depth at the mid-point $(\xi=0.1)$; is centered at $\left(\zeta_{0}, \eta_{0}\right)=(0.25,0.7)$; depth of the crack is nonuniform along the crack: the crack depth decreases from its maximum in the crack center towards the two crack tips, and vanishes at the crack tips (Figure 1). The procedure described in Refs. [1823 ] is carried out to analytically generate mode shapes governed by half-wave numbers $m$ and $n$, 
each consisting of $101 \times 101$ sampling points.

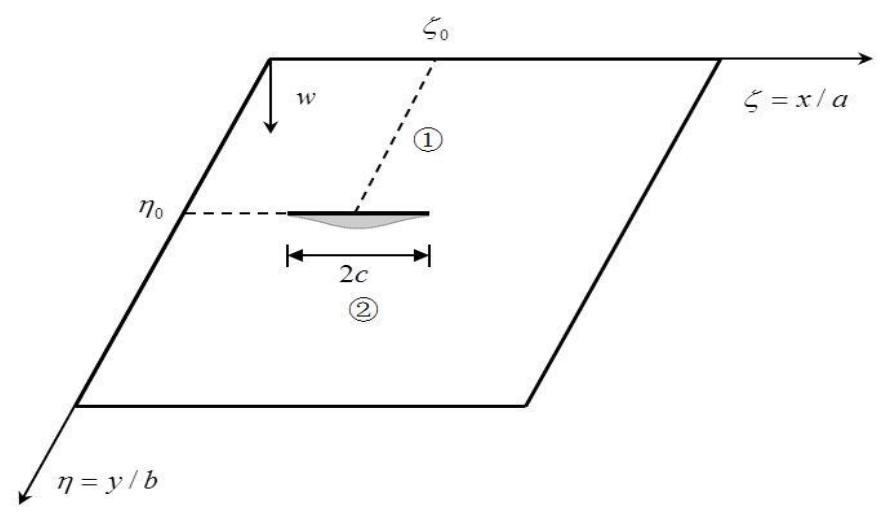

Figure 1. Rectangular plate with non-uniform crack whose depth is represented in gray.

\section{$\mathrm{MC}^{2 \mathrm{D}}$}

The fundamental mode shape with $m$ and $n$ both being 1 is shown in Figure 2. The $\nabla^{2} W$ is obtained by Eq. (2), and shown in Figure 3. In this figure, it can be seen that in the $\nabla^{2} W$, a slim peak appears at the crack location, but nearly masked by the global trends of the $\mathrm{MC}^{2 \mathrm{D}}$, it is unreliable for identifying the crack in the plate. As stated in the introduction, lack of sensitivity to incipient small-scale damage is acknowledged as the first deficiency of the $\mathrm{MC}^{2 \mathrm{D}}$ method [16]. To simulate an actual normal noisy measurement condition, white Gaussian noise is incorporated to produce the noisy mode shape of $80 \mathrm{~dB}$ signal-to-noise (SNR). In the noise-contaminated $\mathrm{MC}^{2 \mathrm{D}}$ (Figure 4), it can be seen that intensive noise totally obliterates actual crack features in $\nabla^{2} W$. Consequently, the $\mathrm{MC}^{2 \mathrm{D}}$ method is unable to identify the crack under such a normal noisy condition. Thus, susceptibility to noise is acknowledged as the second deficiency of the MC ${ }^{2 \mathrm{D}}$ method [16].

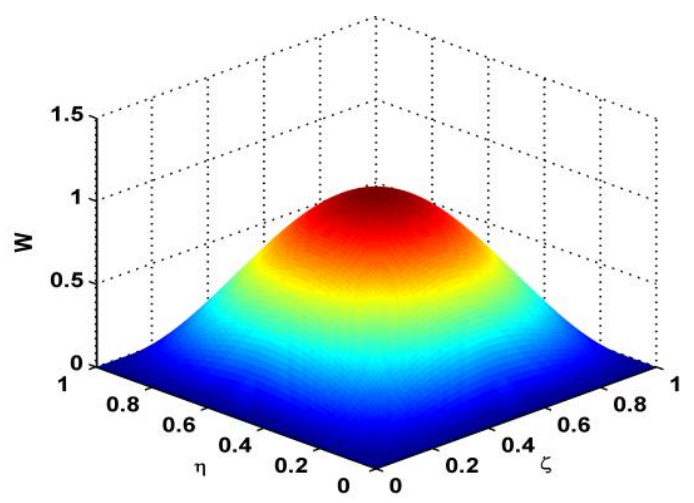

Figure 2. Fundamental mode shape of plate.

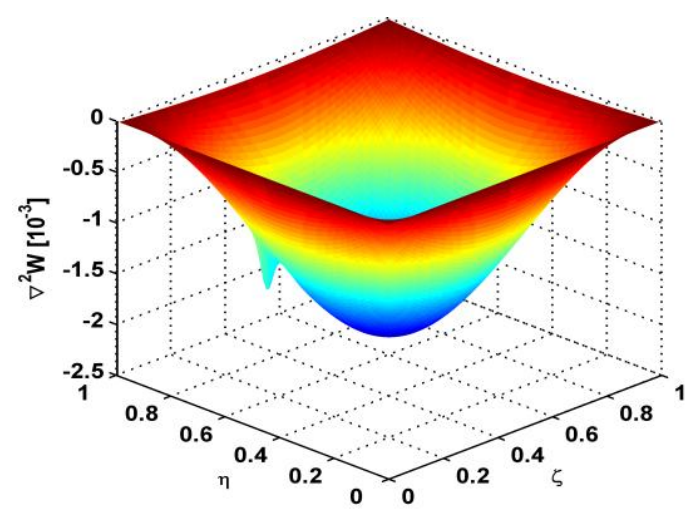

Figure 3. $\mathrm{MC}^{2 \mathrm{D}}$ for the fundamental mode shape under noise-free condition. 


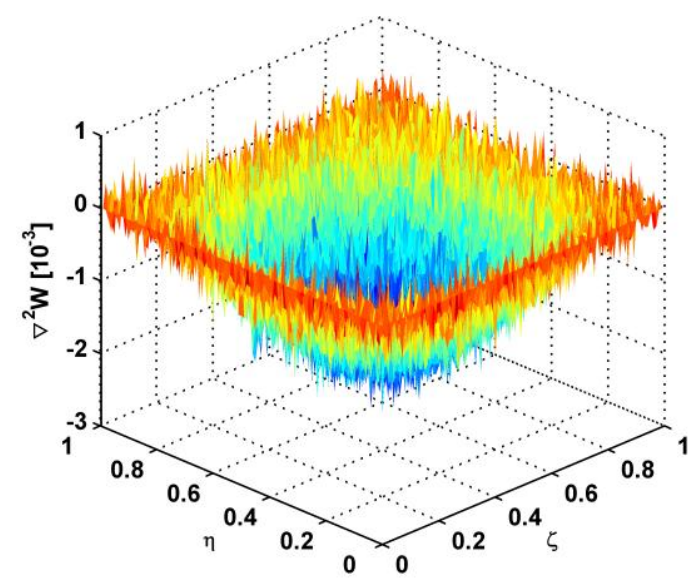

Figure 4. $\mathrm{MC}^{2 \mathrm{D}}$ for the fundamental mode shape under noisy condition.

\section{RW-MC $\mathbf{M C}^{2 \mathrm{D}}$}

The $W_{s}^{\mathrm{Re}^{*}}$ is obtained by Eq. (3) with the $g(x)$ being the Gaussian function. By increasing the parameter of scale up to $s=4$, noise interference in $W_{4}^{\mathrm{Re}^{*}}$ (Figure 5) is significantly reduced. In contrast to the $\nabla^{2} W$ (Figure 4), the RW-MC ${ }^{2 \mathrm{D}}$ can effectively eliminate the noise interference, largely overcoming the susceptibility to noise interference of the $\mathrm{MC}^{2 \mathrm{D}}$. However, it is evident in Figure 5 that the crack-caused features in the $W_{s}^{\mathrm{Re}^{*}}$ are still not prominent relative to its global trends. Hence, the deficiency in the $\mathrm{MCs}^{2 \mathrm{D}}$ (Figure 3) of insensitivity to small damage is still retained in the RW-MC ${ }^{2 \mathrm{D}}$.

Noticeably, abnormal wavelet coefficients occur near the boundaries of $W_{s}^{\mathrm{Re}^{*}}$, referred to as the boundary effect of the WT on a signal with finite length. Interpolation [24] and padding [25] methods are two types of manipulation to deal with this boundary effect. For simplicity in practice, another simple type of manipulation of vanishing abnormal wavelet coefficients $[16,26]$ is employed in this study.

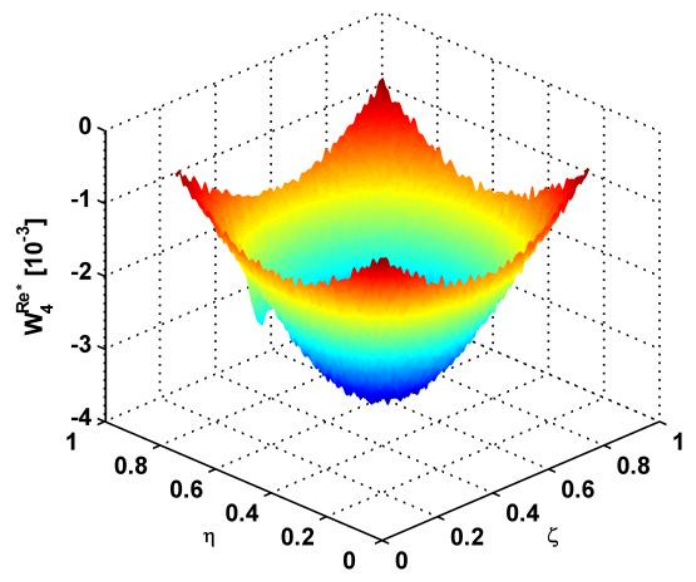

Figure 5. RW-MC ${ }^{2 \mathrm{D}}$ for the fundamental mode shape.

\section{$\mathrm{CW}-\mathrm{MC}^{2 \mathrm{D}}$}

The $W_{s}^{\text {Com* }^{*}}$ is obtained by Eq. (3) with the $g(x)$ being the Gabor function. By increasing the parameter of scale up to $s=10$, noise interference in $W_{10}^{\text {com* }^{*}}$ (Figure 6(a)) is significantly reduced. It should be noted that as the $W_{s}^{\mathrm{Com} *}$ is complex, $W_{s}^{\mathrm{Com} *}$ is represented by its modules for display; and wavelet coefficients of $W_{s}^{\mathrm{Com}^{*}}$ near the boundaries are made to vanish. $W_{10}^{\mathrm{Com}^{*}}$ bears a sharply rising singular peak around the crack location, clearly designating the presence of the crack. In its planform (Figure 6(b)), the strip of higher intensity clearly delineates the crack: the identified crack 
is located at $\eta=0.7$, spanning $\zeta=0.2-0.3$, which corresponds to the actual crack location. In contrast to the RW-MC ${ }^{2 \mathrm{D}}$, the $\mathrm{CW}-\mathrm{MC}^{2 \mathrm{D}}$ can not only eliminate noise interference, but also extract more abundant damage features out of its global trends. Furthermore, the trend of change in the CW$\mathrm{MC}^{2 \mathrm{D}}$ well characterizes the trend of change in depth along the crack: the crack depth decreases from its maximum in the crack center towards the two crack tips, and vanishes at the crack tips.

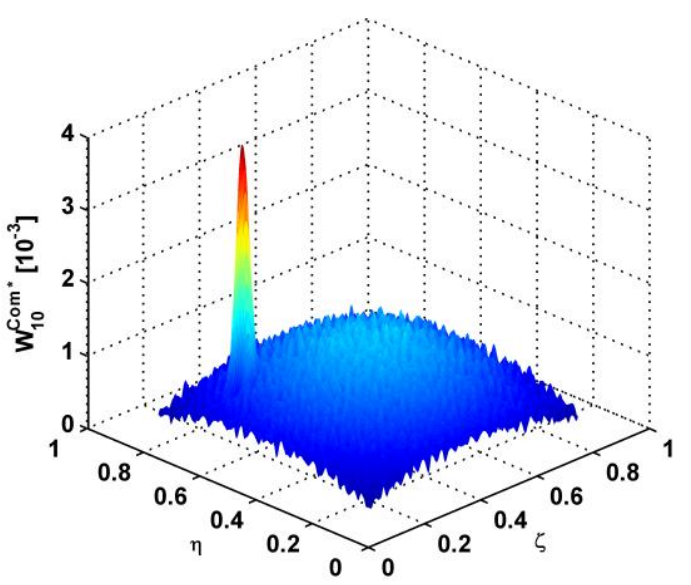

(a)

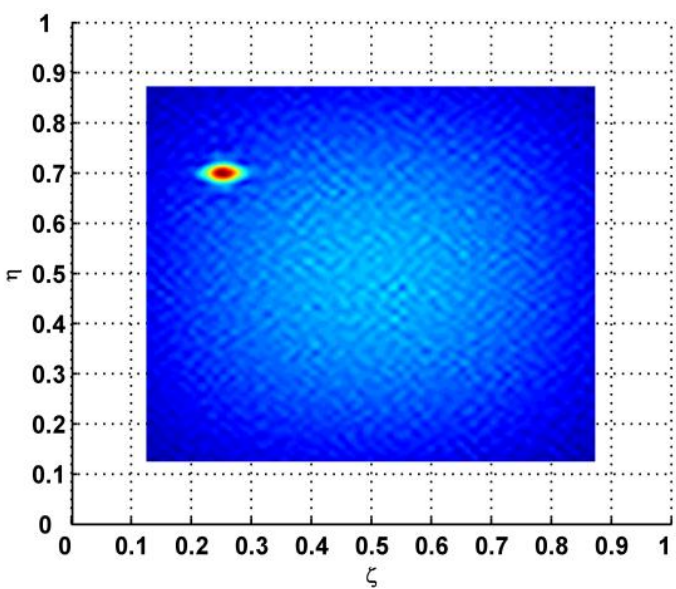

(b)

Figure 6. CW-MC ${ }^{2 \mathrm{D}}$ for the fundamental mode shape (a) and its planform (b).

\section{Conclusions}

Plate-like structural components are essential elements in mechanical, aerospace, and civil engineering structures. In most research on identifying damage in plates, the damage is depicted with a uniform extension depth. On this assumption, various methods for detecting damage in plates have been developed, typically the $\mathrm{MC}^{2 \mathrm{D}}$. However, the extent of extension of actual damage is hardly uniform but gradually reduced from the central point to the edge of the damage, referring to a non-uniform damage. Most traditional methods like the $\mathrm{MC}^{2 \mathrm{D}}$ are not competent to identify such damage. In this study, the $\mathrm{CW}-\mathrm{MC}^{2 \mathrm{D}}$ is created to characterize non-uniform cracks in plates under noisy conditions. The results show that the $\mathrm{CW}-\mathrm{MC}^{2 \mathrm{D}}$ is of solid capability of extracting damage features by eliminating noise interference.

\section{Acknowledgements}

The authors are grateful for the partial support provided by Key Natural Science Foundation of China (No.11132003) and the Fundamental Research Funds for the Key Universities in China (Grant nos. 2014B03914 and 2012B05814).

\section{References}

1. C.R. Farrar, K. Worden, An introduction to structural health monitoring, Philos. T. R. Soc. A., 365, 303-315 (2007).

2. J. Ou, H. Li, Structural Health Monitoring in mainland China: Review and Future Trends, Struct. Health Monit., 9, 219-231 (2010).

3. M.S. Cao, W. Ostachowicz, M. Radzienski, W. Xu, Multiscale shear-strain gradient for detecting delamination in composite laminates, Appl. Phys. Lett., 103, 101910 (2013).

4. S. Wang, Q. Ren, P. Qiao, Structural damage detection using local damage factor, J. Vib. Control, 12, 955-973 (2006). 
5. M. Cao, Z. Su, L. Cheng, H. Xu, A multi-scale pseudo-force model for characterization of damage in beam components with unknown material and structural parameters, J. Sound Vib., 332, 5566-5583 (2013).

6. H. Xu, Z. Su, L. Cheng, J. Guyader, A "Pseudo-excitation" approach for structural damage identification: From "Strong" to "Weak" modality, J. Sound Vib., 337, 181-198 (2015).

7. A. Katunin, Vibration-based spatial damage identification in honeycomb-core sandwich composite structures using wavelet analysis, Compos. Struct., 118, 385-391 (2014).

8. D. Dessi, G. Camerlengo, Damage identification techniques via modal curvature analysis: Overview and comparison, Mech. Syst. Signal Pr., 52, 181-205 (2015).

9. L.H. Yam, Y.Y. Li, W.O. Wong, Sensitivity studies of parameters for damage detection of plate-like structures using static and dynamic approaches, Eng. Struct., 24, 1465-1475 (2002).

10. P. Qiao, K. Lu, W. Lestari, J. Wang, Curvature mode shape-based damage detection in composite laminated plates, Compos. Struct., 80, 409-428 (2007).

11. Y.Y. Li, L. Cheng, L.H. Yam, W.O. Wong, Identification of damage locations for plate-like structures using damage sensitive indices: strain modal approach, Comput. Struct., 80, 1881-1894 (2002).

12. N. Hu, H. Fukunaga, M. Kameyama, Y. Aramaki, F.K. Chang, Vibration analysis of delaminated composite beams and plates using a higher-order finite element, Int. J. Mech. Sci., 44, 1479-1503 (2002).

13. D. Wu, S.S. Law, Damage localization in plate structures from uniform load surface curvature, J. Sound Vib., 276, 227-244 (2004).

14. M.K. Yoon, D. Heider, J.W. Gillespie, C.P. Ratcliffe, R.M. Crane, Local damage detection using the two-dimensional gapped smoothing method, J Sound Vib., 279, 119-139 (2005).

15. Z. Yang, M. Radzienski, P. Kudela, W. Ostachowicz, Two-dimensional modal curvature estimation via Fourier spectral method for damage detection, Compos. Struct., 148, 155-167 (2016).

16. W. Xu, M. Cao, W. Ostachowicz, M. Radzienski, N. Xia, Two-dimensional curvature mode shape method based on wavelets and Teager energy for damage detection in plates, J. Sound Vib., 347, 266-278 (2015).

17. S. Mallat, W.L. Hwang, Singularity detection and processing with wavelets, IEEE Trans. on Inform. Theory, 38, 617-643 (1992).

18. S.E. Khadem, M. Rezaee, An analytical approach for obtaining the location and depth of an allover part-through crack on externally in-plane loaded rectangular plate using vibration analysis, J. Sound Vib., 230, 291-308 (2000).

19. S.E. Khadem, M. Rezaee, Introduction of modified comparison functions for vibration analysis of a rectangular cracked plate, J. Sound Vib., 236, 245-258 (2000).

20. E. Douka, S. Loutridis, A. Trochidis, Crack identification in plates using wavelet analysis, J. Sound Vib., 270, 279-295 (2004).

21. S. Loutridis, E. Douka, L.J. Hadjileontiadis, A. Trochidis, A two-dimensional wavelet transform for detection of cracks in plates, Eng. Struct., 27, 1327-1338 (2005).

22. L.J. Hadjileontiadis, E. Douka, Crack detection in plates using fractal dimension, Eng. Struct., 29, 1612-1625 (2007).

23. H.F. Lam, T. Yin, Application of two - dimensional spatial wavelet transform in the detection of an obstructed crack on a thin plate, Struct. Control and Health Monit., 19, 260-277 (2012). 
24. M. Rucka, K. Wilde, Crack identification using wavelets on experimental static deflection profiles, Eng. Struct., 28, 279-288 (2006).

25. L. Montanari, B. Basu, A. Spagnoli, B.M. Broderick, A padding method to reduce edge effects for enhanced damage identification using wavelet analysis, Mech. Syst. Signal Pr., 52-53, 264-277 (2015).

26. W. Xu, M. Radzienski, W. Ostachowicz, M. Cao, Damage detection in plates using twodimensional directional Gaussian wavelets and laser scanned operating deflection shapes, Struct. Health Monit., 12, 457-468 (2013). 